\title{
Demonstration of Rapid Frequency Selective Reconstruction \\ for Image Resolution Enhancement
}

Nils Genser, Jürgen Seiler, and André Kaup

Multimedia Communications and Signal Processing

Friedrich-Alexander-Universität Erlangen-Nürnberg (FAU), Cauerstr. 7, 91058 Erlangen, Germany

\section{Motivation}

- Many scenarios, where amplitude information of an image is not available on a regular, rectangular grid - Optical Cluster Eye

Micro-Optical Artificial Compound Eyes

- Reducing visible influence of aliasing

Super-Resolution techniques [1]

- For further processing or displaying, a resampling to the full regular grid is required
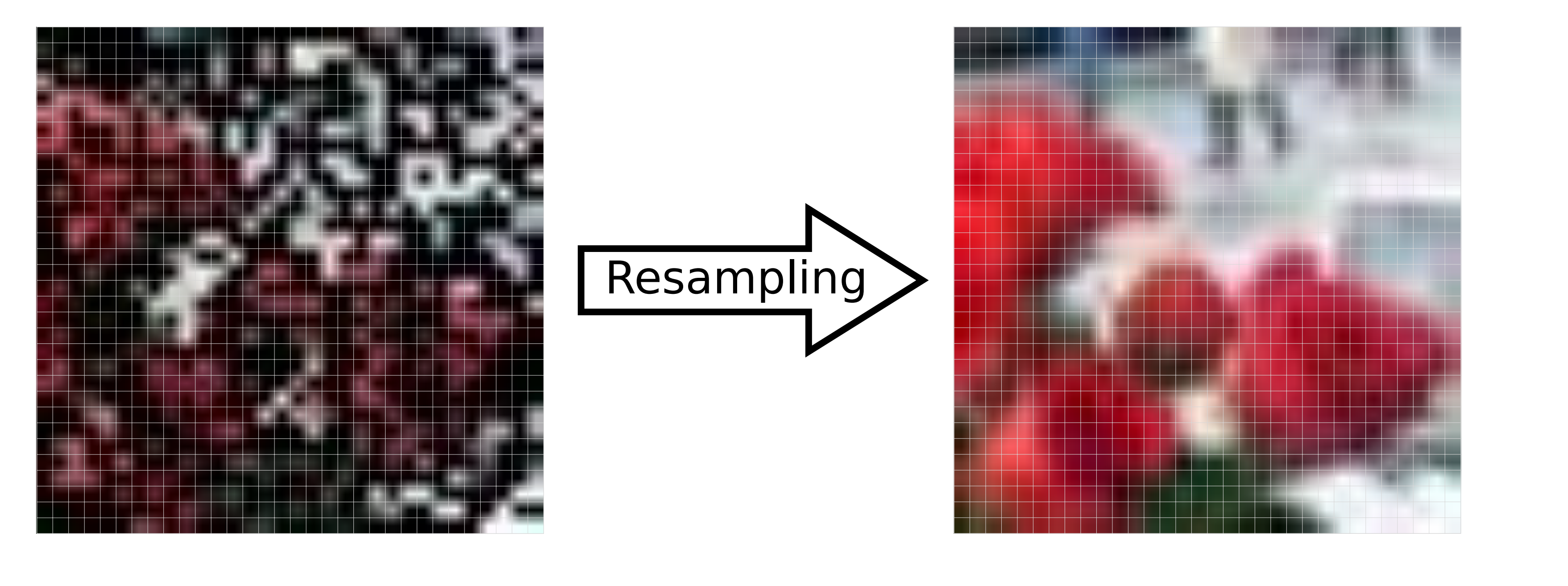

\section{Frequency Selective Reconstruction (FSR) [2]}

- Iterative sparse model generation by superimposing weighted Fourier basis functions: $g[m, n]=\sum_{k \in \mathcal{K}} \hat{c}_{k} \varphi_{k}[m, n]$

- Spatial weighting function

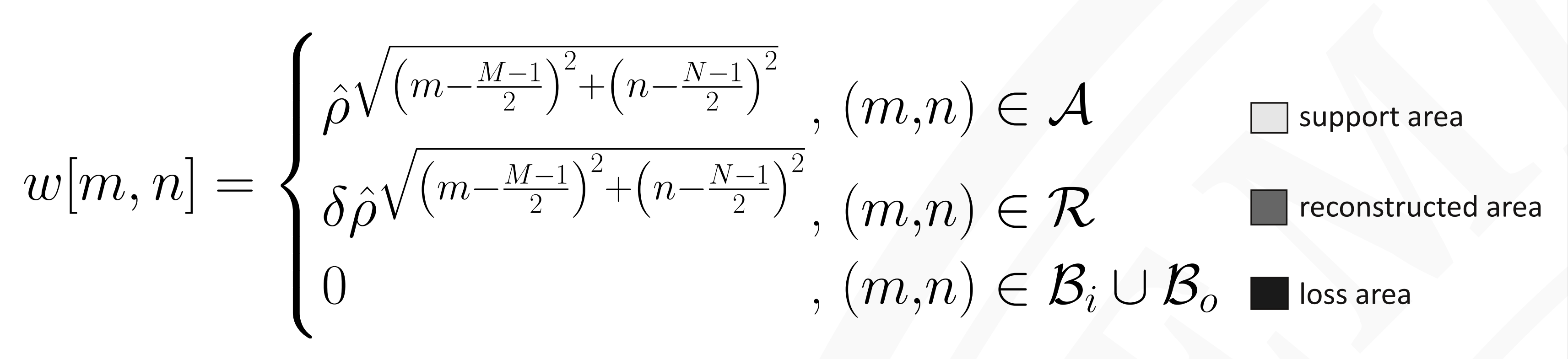

- Fixed frequency prior to favor low frequencies

$$
w_{f}[k, l]=\left(1-\sqrt{2} \sqrt{\frac{\tilde{k}^{2}}{M^{2}}+\frac{\tilde{l}^{2}}{N^{2}}}\right)^{2}
$$

$\rightarrow$ Approximation of the Optical Transfer Function

\begin{tabular}{|c|c|c|c|}
\hline \multicolumn{4}{|c|}{ 3. Demonstration } \\
\hline \multirow{9}{*}{$\begin{array}{l}\text { Algorithmic Enhancements } \\
\text { - YUV color space dependent reconstruction [3] } \\
\text { - Linear interpolation for chroma channels } \\
\text { - FSR for luminance channel, only } \\
\text { - Restrict number } \\
\text { and set of basis } \\
\text { functions [4], [5] } \\
\text { - Real-valued, } \\
\text { spectrally constrained model [6] } \\
\text { - Texture dependent block partitioning and } \\
\text { parameter estimation [7] }\end{array}$} & \multirow{9}{*}{$\begin{array}{l}\text { Software Optimizations } \\
\text { - C++ implementation using FFTW3 library } \\
\text { - Parallelization using OpenMP pragmas to } \\
\text { make use of all processor cores } \\
\text { - Vectorization } \\
\text { - Manual preparation of loops } \\
\rightarrow \text { Avoid control flows } \\
\text { - GCC's autovectorization features } \\
\text { and OpenMP's SIMD pragmas }\end{array}$} & \multicolumn{2}{|c|}{ Test Systems } \\
\hline & & Notebook & \\
\hline & & & $i 7-6700 \mathrm{HQ}$ \\
\hline & & Speed & $2.60 \mathrm{GHz}$ \\
\hline & & & \\
\hline & & Xeon & \\
\hline & & & $2 \times$ Xeon E5-2630v4 \\
\hline & & Speed & $2.20 \mathrm{GHz}$ \\
\hline & & RAM & $32 \mathrm{~GB}$ \\
\hline
\end{tabular}

$\rightarrow$ Improved reconstruction quality

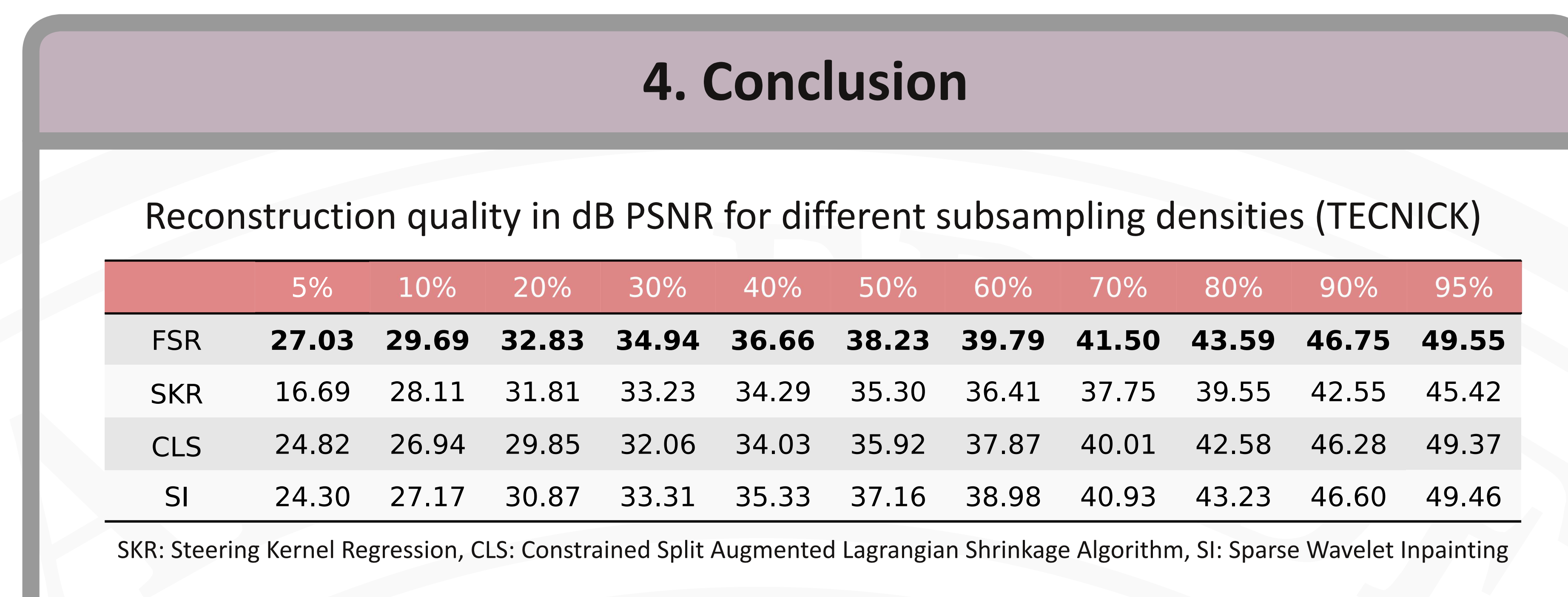

- FSR well suited for image reconstruction problems

- Visually noticeable gains of several dB PSNR compared

to other state-of-the-art algorithms

- Acceleration of the reconstruction process by

$\rightarrow$ Reducing the number of basis functions

$\rightarrow$ Lowering the required set of basis functions

$\rightarrow$ Texture dependent block partitioning

$\rightarrow$ Partitioning based parameter estimation

$\rightarrow$ Setting up a real-valued, spectrally constrained model

$\rightarrow$ Software optimizations

Accelerated reconstruction process:

$\rightarrow 15$ fps using test system Notebook

$\rightarrow 25 \mathrm{fps}$ for test system Xeon

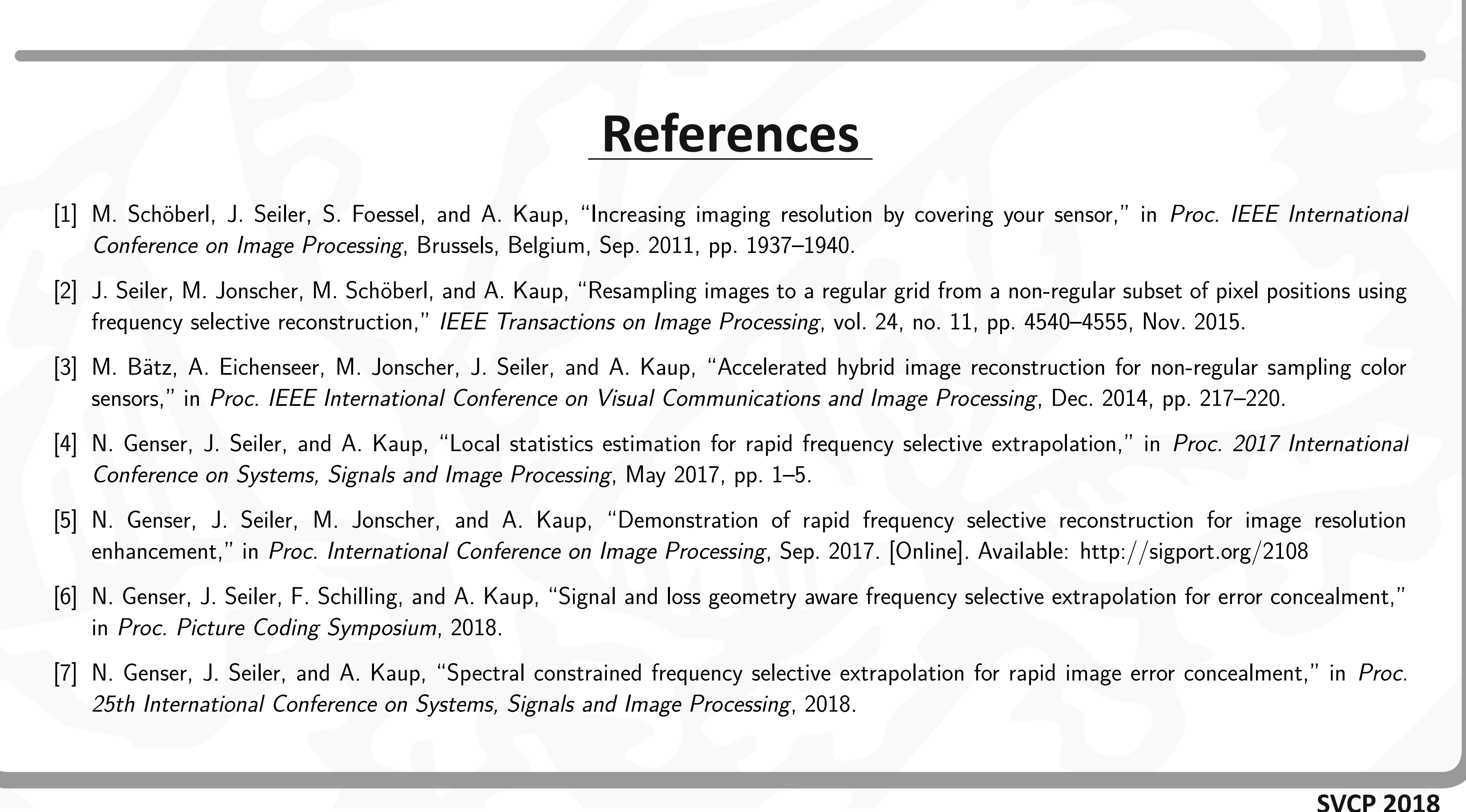

